\title{
Structural evolution in bulk metallic glass under high-temperature tension
}

X. D. Wang, H. B. Lou, J. Bednarcik, H. Franz, H. W. Sheng, Q. P. Cao, and J. Z. Jiang

Citation: Appl. Phys. Lett. 102, 051909 (2013); doi: 10.1063/1.4790393

View online: https://doi.org/10.1063/1.4790393

View Table of Contents: http://aip.scitation.org/toc/apl/102/5

Published by the American Institute of Physics

\section{Articles you may be interested in}

Atomic structure of binary $\mathrm{Cu}_{64.5} \mathrm{Zr}_{35.5}$ bulk metallic glass

Applied Physics Letters 92, 011902 (2008); 10.1063/1.2828694

Pressure effects on structure and dynamics of metallic glass-forming liquid

The Journal of Chemical Physics 146, 024507 (2017); 10.1063/1.4973919

Alloying strongly influences the structure, dynamics, and glass forming ability of metallic supercooled liquids Applied Physics Letters 93, 111913 (2008); 10.1063/1.2987727

Structural crossover in a supercooled metallic liquid and the link to a liquid-to-liquid phase transition Applied Physics Letters 108, 211907 (2016); 10.1063/1.4952724

Kinetic and thermodynamic studies of the fragility of bulk metallic glass forming liquids Journal of Applied Physics 108, 063501 (2010); 10.1063/1.3480805

Local strain behavior of bulk metallic glasses under tension studied by in situ $\mathrm{x}$-ray diffraction Applied Physics Letters 94, 011911 (2009); 10.1063/1.3064136

\section{Instruments for Advanced Science}
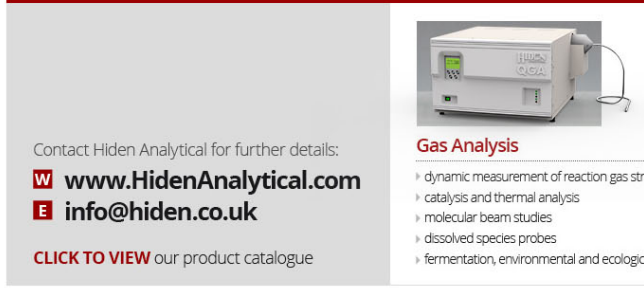

Gas Analysis

dynamic measurement of reaction gasstreams

c cataysis and thermal analysis

molecuar beam studes

dissolved species probes

fermentation, envirormental and ecological studi

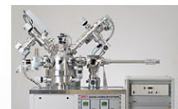

Surface Science

UHVTPD

, end point detection in in beam etch

, elemental imaging-sufface mapping

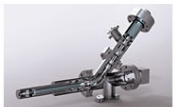

Plasma Diagnostics

, plasma source characterization etch and deposition process reaction kinetic studies

analysis of neutral and radical species



Vacuum Analysis partial pressure measurement and control of process gases , reactive sputter process control vacuum diagnostic 


\title{
Structural evolution in bulk metallic glass under high-temperature tension
}

\author{
X. D. Wang, ${ }^{1, a)}$ H. B. Lou, ${ }^{1}$ J. Bednarcik, ${ }^{2}$ H. Franz, ${ }^{2}$ H. W. Sheng, ${ }^{3}$ Q. P. Cao, ${ }^{1}$ \\ and J. Z. Jiang ${ }^{1, a)}$ \\ ${ }^{1}$ Department of Materials Science and Engineering, International Center for New-Structured Materials \\ (ICNSM) and Laboratory of New-Structured Materials, Zhejiang University, Hangzhou 310027, \\ People's Republic of China \\ ${ }^{2}$ Hamburger Synchrotronstrahlungslabor am Deutschen Elektronen Synchrotron, Notkestrasse 85, \\ D-22603 Hamburg, Germany \\ ${ }^{3}$ School of Physics, Astronomy and Computational Sciences, George Mason University, Fairfax, \\ Virginia 22030, USA
}

(Received 2 November 2012; accepted 22 January 2013; published online 5 February 2013)

\begin{abstract}
The tensile behavior of a $\mathrm{Cu}_{46} \mathrm{Zr}_{46} \mathrm{Al}_{8}$ bulk metallic glass (BMG) at elevated temperatures has been studied using in situ $\mathrm{x}$-ray diffraction and molecular dynamics simulation. It is demonstrated that excess open volume is generated during elastic deformation and accumulated enough before plastic flow starts. The open volume almost keeps constant during homogeneous deformation, suggesting that a high content of open volume is a key point for developing BMGs with pronounced tensile plasticity. (C) 2013 American Institute of Physics. [http://dx.doi.org/10.1063/1.4790393]
\end{abstract}

As a class of metallic materials, bulk metallic glasses (BMGs) have received much attention due to their superior mechanical, physical, and chemical properties compared to their crystalline counterparts. ${ }^{1,2}$ However, one major drawback of metallic glasses, preventing their applications as structural materials, is their brittleness. At room temperature, plastic deformation of large-sized BMGs is highly localized into narrow shear bands, which usually propagate across the sample rapidly, resulting in catastrophic failure upon tension. ${ }^{3}$ However, with increasing temperature, BMG samples can undergo transitions from localized shear deformation, non-Newtonian necking and to homogeneous Newtonian flow. ${ }^{4,5}$ Near-net-shape fabrications can be realized via homogeneous flow, which is promising for microelectromechanical system (MEMS) devices. ${ }^{6}$ A recent study showed that metallic glasses at room temperature can be perceived as a mixture consisting of tightly bonded atomic clusters and loosely bonded free volume regions. ${ }^{7-9}$ In the elastic regime, atomic clusters undergo elastic deformation, and free-volume regions behave like supercooled liquids. At high temperatures, viscous flow becomes dominant. How the atomic structural evolution happens upon deformation has never been reported experimentally. In this study, we report on the tensile behavior of a $\mathrm{Cu}_{46} \mathrm{Zr}_{46} \mathrm{Al}_{8}$ BMG using in situ high temperature high-energy $\mathrm{x}$-ray diffraction (HEXRD) combined with molecular dynamics (MD) simulation. Atomic structure evolution from elastic to plastic deformation for a $\mathrm{Cu}_{46} \mathrm{Zr}_{46} \mathrm{Al}_{8}$ BMG under tension has been experimentally and theoretically investigated.

Using high purity raw materials, pre-alloyed ingots of nominal composition $\mathrm{Cu}_{46} \mathrm{Zr}_{46} \mathrm{Al}_{8}$ were prepared by arc melting under a Ti-gettered purified argon atmosphere. Each alloy ingot of about $15 \mathrm{~g}$ was remelted several times to improve the homogeneity. From ingots, sheet-shaped samples $1 \mathrm{~mm}$ thick, $10 \mathrm{~mm}$ wide, and $60 \mathrm{~mm}$ long were prepared by suction

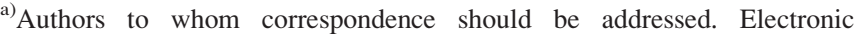
addresses: wangxd@zju.edu.cn and jiangjz@zju.edu.cn.
}

casting in a water-cooled copper mold. Fig. 1 schematically shows the experimental setup for in situ tensile measurement at the BW5 station of HASYLAB, Hamburg. The details can be found in Refs. 10 and 11. For high temperature tension, a small heater was mounted beneath the sample, in which a hole of diameter $3 \mathrm{~mm}$ was fabricated to let x-ray pass through. The temperature was detected by a thermocouple mounted at a position near the middle between the sample and the heater. Prior to tension, the temperature was set to the given value, and then the tension started at a strain rate of about $5 \times 10^{-4} \mathrm{~s}^{-1}$. The beam size and wavelength used were $0.5 \times 0.5 \mathrm{~mm}^{2}$ and $0.124 \AA$, respectively. The XRD patterns were recorded by a flat panel Si detector (Perkin Elmer 1621) with $200 \times 200 \mu \mathrm{m}^{2}$ pixel size and $2048 \times 2048$ pixels. Exposure time was $2 \mathrm{~s}$, and 5 diffraction patterns were summed for each data set. From the record of time versus elongation and load by the software of the tensile module, the engineering stress and strain were estimated.

Using newly developed embedded atom method (EAM) potentials ${ }^{12}$ for the $\mathrm{Cu}-\mathrm{Zr}$-Al system from $a b$ initio calculations, the tensile behavior of this BMG at $723 \mathrm{~K}$ was



FIG. 1. Layout of the experimental setup for in situ tension measurement. The sample and detector are perpendicular to the incident $\mathrm{x}$-ray beam. 
simulated using the LAMMPS code. ${ }^{13}$ A small box (10000 atoms) with three dimensional (3D) periodic boundary conditions (PBCs) was heated to $2200 \mathrm{~K}$ and equilibrated for 2 ns (time step $2 \mathrm{fs}$ ), and then quenched at a cooling rate of 2.5 $\times 10^{12} \mathrm{~K} / \mathrm{s}$ to the glassy state at zero external pressure (NPT ensemble and Nose-Hoover thermostat). For the uniaxial tension, a large sample (about $22.6 \mathrm{~nm} \times 11.5 \mathrm{~nm} \times 67.4 \mathrm{~nm}$ ) containing 960000 atoms was constructed by replicating the 10000-atom configuration in 3D space, which were then annealed for $1 \mathrm{~ns}$ at $723 \mathrm{~K}$. PBCs were imposed in Z-direction, while free surfaces were used in the $\mathrm{X}$ - and Y-directions to allow necking at free surfaces. Uniaxial tension was applied along the $\mathrm{Z}$-direction to move atoms to two sides at a constant strain rate of $10^{8} \mathrm{~s}^{-1}$.

The glass transition temperature $T_{g}$ for $\mathrm{Cu}_{46} \mathrm{Zr}_{46} \mathrm{Al}_{8}$ BMG measured by differential scanning calorimeter (DSC) is $716 \mathrm{~K}$ at a heating rate of $20 \mathrm{~K} / \mathrm{min}$. In this work, tensile measurements at temperatures of $298 \mathrm{~K}, 693 \mathrm{~K}$, and $723 \mathrm{~K}$ have been performed. Fig. 2(a) shows the engineering stressstrain curves (solid lines) for the samples measured at $298 \mathrm{~K}$, $693 \mathrm{~K}$, and $723 \mathrm{~K}$, respectively. At $298 \mathrm{~K}$, the sample exhibits almost perfect elastic behavior followed by catastrophic failure with a tensile strength of $1800 \mathrm{MPa}$ and an elastic strain of $1.66 \%$. With increasing temperature, the strength decreases and plasticity increases obviously. Using XRD, the angular dependent strain can be calculated from the position
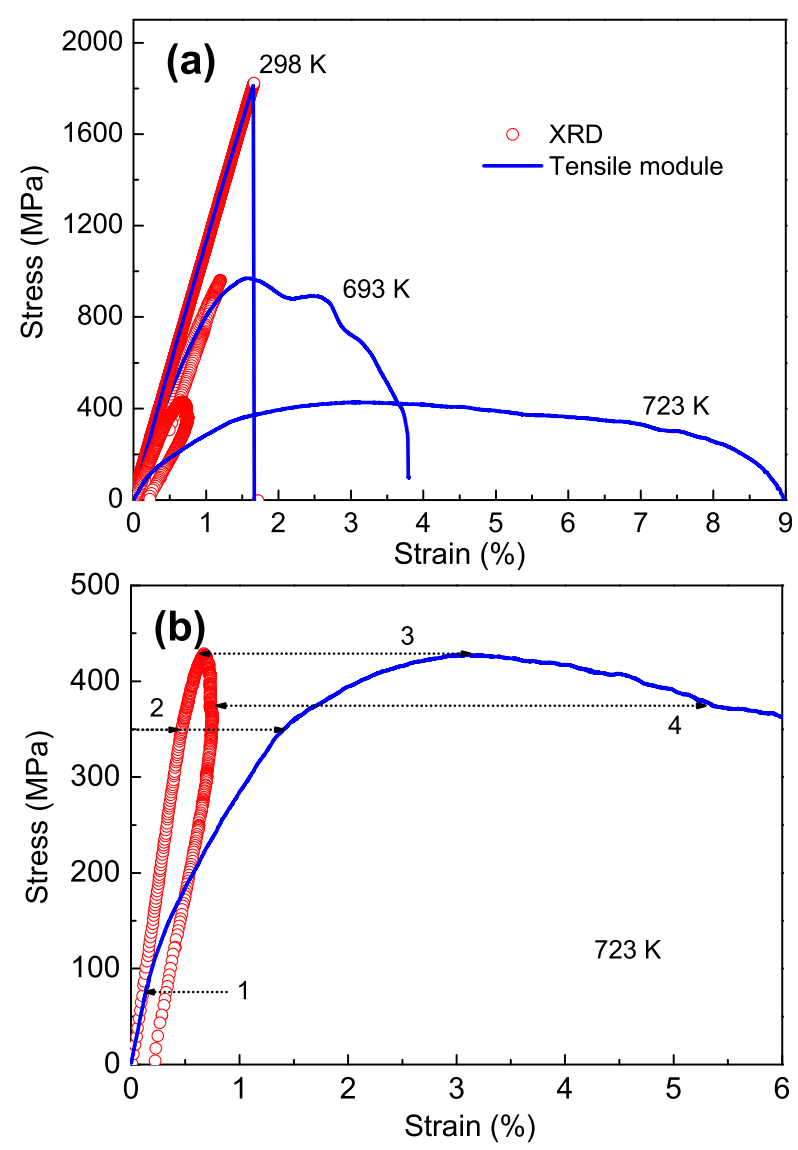

FIG. 2. (a) Engineering stress-strain curves of $\mathrm{Cu}_{46} \mathrm{Zr}_{46} \mathrm{Al}_{8}$ BMG measured by a tensile module (solid line) and by XRD (open circle) at $298 \mathrm{~K}, 693 \mathrm{~K}$, and $723 \mathrm{~K}$. (b) The local magnification of the stress-strain curves determined by $\mathrm{XRD}$ and tensile module at $723 \mathrm{~K}$, showing the distinct difference between them. shift of the first maximum in $S(q),{ }^{14,15} \varepsilon\left(\sigma, \eta_{i}\right)=\left(q_{1}\left(\sigma, \eta_{i}\right)\right.$ $\left.-q_{1}\left(0, \eta_{i}\right)\right) / q_{1}\left(\sigma, \eta_{i}\right)$, where $q_{1}$ is the first-peak position in $S(q)$ under stress $\sigma$, and $\eta_{i}$ is the angle between the tensile stress and the strain, $i$ is an integer running from 0 to 35 . The strains in the tensile direction $\varepsilon_{11}$ and transverse direction $\varepsilon_{22}$ under stress $\sigma$ were obtained by fitting $\varepsilon(\sigma, \eta)$ with the equation: $\varepsilon(\sigma, \eta)=\varepsilon_{11} \cos ^{2} \eta+\varepsilon_{12} \sin \eta \cos \eta+\varepsilon_{22} \sin ^{2} \eta .^{12} \quad$ By analyzing the shift of the structure factor maximum in the XRD patterns, only the change of the average interatomic distance of nearest neighbors can be estimated, from which the elastic moduli and Poisson's ratios are obtained to be $109 \mathrm{GPa}$ and 0.34 at $298 \mathrm{~K}, 80 \mathrm{GPa}$ and 0.38 at $693 \mathrm{~K}$, and $77 \mathrm{GPa}$ and 0.46 at $723 \mathrm{~K}$ in the elastic regime, respectively. The values are close to $96.4 \mathrm{GPa}$ and 0.368 measured by ultrasonic method for this alloy at room temperature. ${ }^{16}$ In contrast, the strain measured by the tensile module contains pure elastic, anelastic, and even plastic strain when plastic flow starts. As shown in Fig. 2(a), the tensile strain $\varepsilon_{11}$ obtained from XRD (open circles) within the whole tension process follows the trace measured by the tensile module at $298 \mathrm{~K}$. However, they obviously deviate from each other when tensions are performed at high temperatures. Thus, at high temperatures the strain determined by XRD obviously differs from that measured by the tensile module. Explanations are given in the following sections.

Fig. 2(b) shows the tensile curves measured at $723 \mathrm{~K}$. Obviously, the behavior can be subdivided into four regions. The strains measured by both methods almost overlap in the elastic regime (below stress 1 (about $75 \mathrm{MPa}$ ) or strain of about $0.13 \%$ ), but deviate from each other above stress 1 . In the region between stress 1 and stress 2 (about $350 \mathrm{MPa}$ ), the strain measured by the tensile module significantly increases from about $0.13 \%$ to $1.4 \%$. However, the strain measured by XRD still increases linearly with stress from about $0.13 \%$ to $0.46 \%$. The difference in strain estimated by both methods indicates anelastic strain exists. At stresses above stress 2, the strain measured by the tensile module increases largely (from $1.4 \%$ to about $3 \%$ ), whereas the strain measured by XRD goes almost linearly (from $0.47 \%$ to about $0.7 \%$ ) with stress up to stress 3 ( $427 \mathrm{GPa}$ ) having slightly reduced slope. It suggests that anelastic strain in this regime (from stress 1 to stress 3) promotes the macroscopic elongation of the sample by forming open volumes (free volume and nano-voids), which has little effect on XRD patterns because of almost negligible x-ray scattering of nano-voids. After the stress reaching the maximum of about $427 \mathrm{MPa}$, it is interesting to find that as the stress decreases slightly from $427 \mathrm{MPa}$ to $375 \mathrm{MPa}$ (stress 4), the strain determined by XRD almost retains constant (from $0.7 \%$ to $0.73 \%$ ) while the strain measured by the tensile module increases from about $3 \%$ to $5.4 \%$. After stress 4 , necking occurs since the strain measured by the tensile module increases from about $5.4 \%$ to $9 \%$, while the engineering stress gradually decreases to zero. In this region, the strain determined by XRD almost linearly recovers with decreasing the engineering stress. At the end, there still remains a value of about $0.23 \%$ strain (determined by XRD) just before fracture.

MD simulations play an important role in understanding the deformation behavior of BMGs on the atomic level. Simulations predicted the intrinsic plasticity of MGs, ${ }^{17}$ the 
shear-band behavior, ${ }^{18}$ and uncovered the atomic configuration change in MGs upon deformation. ${ }^{19}$ Thus, to unravel local atomic configuration changes in BMGs upon deformation at high temperatures, we carried out MD simulation for the BMG at $723 \mathrm{~K}$. Fig. 3(a) shows the comparison between the structure factors $S(q)$ measured by XRD and calculated from the MD simulation. The simulated $S(q)$ is calculated from about 6950 atoms extracted by a cubic box with edge length of $50 \AA$. Inset is the pair correlation function $g(r)$ obtained by XRD and MD simulation. Agreements in $S(q)$ and $g(r)$ between the XRD results and MD calculations confirm that the constructed model for this BMG used here is reasonable. Fig. 3(b) shows the engineering stress-strain curves of the alloy tensioned at $723 \mathrm{~K}$ by MD simulation. Although large strain is achieved (most likely due to smallsized sample and homogeneous temperature field in MD case), the sample tensioned at $723 \mathrm{~K}$ by MD captures almost all experimentally observed features for the bulk sample in Fig. 2(b). A linear elastic region occurs in the strain range of $0 \%-0.7 \%$. Strain further increases with stress after $0.7 \%$, but having lower slopes. At the strain value of about $5 \%$, the stress reaches the maximum and then decreases very slightly with strain up to $50 \%$. The inset shows snapshots for the sample at different strains. Above 50\% strain, the decrease in stress in much steeper until fracture occurs at around $100 \%$ strain.


FIG. 3. (a) Comparisons in structure factors $S(q)$ and (inset) pair correlation function $g(r)$ measured by XRD and by MD simulation for the as-cast sample at $723 \mathrm{~K}$. (b) The tensile stress-strain curve simulated by MD for the sample at $723 \mathrm{~K}$. Inset of (b) shows snapshots of the sample during tensile test at $723 \mathrm{~K}$.
Open (or free) volume has been considered to be closely related to the plastic flow of BMGs. ${ }^{20}$ One general view is that the more open volume exists, the more easily the plastic deformation happens. ${ }^{21}$ How open volume does change upon deformation is still a debated issue. ${ }^{22-24} \mathrm{~A}$ latest MD simulation reported that two different cavitation behaviors happened in one brittle and another ductile MG upon fracture. ${ }^{25}$ Using the atomic configurations constructed by MD, we further approximately evaluate the open volume change by calculating the atomic number change in a fixed $80 \times 80 \times 300$ $\AA^{3}$ rectangular box, namely, $\Delta V=V / N \times\left(N_{0}-N\right)$, where $N_{0}$ and $N$ are the number of atoms at the strain of zero and $\varepsilon$, $\mathrm{V}$ is the box volume. As shown in Fig. 4(a), after the elastic region (about $0.7 \%$ strain), open volume quickly increases with strain up to about $1.5 \%$, and then slowly increases until the starting of plastic flow at about 5\% strain. With further increasing strain up to about $10 \%$, the open volume almost keeps a constant value. When the strain is beyond $10 \%$, the open volume change shows a trend of slight decrease, predicting that the necking process starts. For elastic deformation, the volume expansion can be calculated using $\Delta V / V$ $=(1-2 \nu) \varepsilon, \nu$ is the Poisson's ratio and $\varepsilon$ is the magnitude of elastic strain. The Poisson's ratio is approximately estimated to be about 0.48 in the elastic regime by checking the strain change in the transverse direction against that in the tensile direction, which is slightly larger than the experimental value 0.46 . The $\Delta V / V$ linearly changing with the $\varepsilon$ is also plotted in Fig. 4(a). At strain of $0.7 \%, \Delta V / V=0.025 \%$. By extrapolating this line, one can find that within the strain range from $0.7 \%$ to $7 \%$, the pure elastic-induced volume expansion is much less than the value predicted by MD



(b)

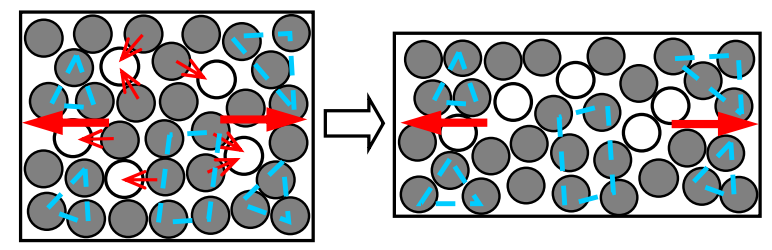

FIG. 4. (a) Calculated open volume change for $\mathrm{Cu}_{46} \mathrm{Zr}_{46} \mathrm{Al}_{8} \mathrm{BMG}$ tensioned at different strains at $723 \mathrm{~K}$ using MD produced atomic configurations and pure elastic deformation. The MD simulated stress-strain curve at $723 \mathrm{~K}$ is also plotted for comparison. (b) 2-dimentional schematic illustration of sample extension with rearrangement of open volumes or loosely packed regions labeled by open circles and tightly bonded regions labeled by dashed lines in the plastic flow at $723 \mathrm{~K}$. 
simulation. Thus, although the elastic-induced volume expansion can make open volume increase to some extent, more open volumes are indeed created after $0.7 \%$ strain and reach a maximum level of about $0.3 \%$ at a strain of about $4 \%$, which do, most likely, not distribute in a narrow region, such as shear bands. If perceiving metallic glasses as a mixture consisting of atomic clusters and open volume regions, ${ }^{7}$ it is not unreasonable to consider that the fraction of atomic clusters (or atoms) in the vicinity of open volumes, which are loosely bonded, is relatively high at the strain of about $4 \%$. Upon further tension, such atomic clusters (or atoms) could be jumped into the tensile direction. In other words, open volumes in the sample could be rearranged during further tensile deformation while their content does not increase. Consequently, these movements of atomic clusters (or atoms) and open volumes directly result in large macroscopic elongation of the sample and plastic deformation. Fig. 4(b) schematically displays plastic elongation in BMGs via the rearrangement of open volumes.

The results obtained by MD simulation almost capture the major features on the stress-strain curve of above experimental measurement. Based on both experimental and theoretical results for the $\mathrm{Cu}-\mathrm{Zr}-\mathrm{Al} \mathrm{BMG}$ obtained here during tension, three striking features can be deduced: (1) open volumes in the sample increase quickly after pure elastic deformation; (2) high content of open volume is the prerequisite for tensile plastic deformation of MGs; and (3) during plastic deformation, the content of open volume does not increase, but open volumes could be rearranged under stress so that macroscopic elongation of the sample and necking happens. We believe that for ductile BMGs, open volumes should be easily created and rearranged during deformation in the whole sample, rather than only in a narrow regime, e.g., shear bands. These results will be useful for understanding deformation behavior of BMGs and small-sized MGs, and for designing ductile BMGs at ambient condition.

In summary, the atomic structure evolution is in situ experimentally studied for a $\mathrm{Cu}_{46} \mathrm{Zr}_{46} \mathrm{Al}_{8}$ BMG under tension at elevated temperatures. By combining XRD data with MD simulation, we find that excess open volumes are generated before plastic flow starts and almost keep constant in plastic deformation. The plastic deformation of MGs can be regarded as the rearrangement of atomic clusters (or atoms) which becomes possible after the creation of high content of excess open volumes. In addition, the average interatomic distance between the nearest neighbors determined by XRD increases with strain before plastic deformation at $723 \mathrm{~K}$ but changes little in the plastic flow before necking.
Authors would like to thank the HASYLAB staff of BW5 at Hamburg, Germany for assistance during the measurements. Financial supports from the NSFC (Grant Nos. 51071141, 51010006, 60776014, 60876002, 10804096, 50920105101, and 10979002), National Key Basic Research Program of China (973 Program, 2012CB825700), Zhejiang University-Helmholtz cooperation fund (IK Ch-0002), the fundamental research funds for the central Universities, the Department of Science and Technology of Zhejiang province and Zhejiang University are gratefully acknowledged. The magic supercomputer in Shanghai Supercomputer Center (SSC) is also acknowledged for supporting the MD simulations.

\footnotetext{
${ }^{1}$ A. Inoue, Acta Mater. 48, 279 (2000).

${ }^{2}$ W. L. Johnson, MRS Bull. 24, 42 (1999).

${ }^{3}$ C. A. Schuh, T. C. Hufnagel, and U. Ramamurty, Acta Mater. 55, 4067 (2007).

${ }^{4}$ Y. Kawamura, T. Shibata, A. Inoue, and T. Masumoto, Appl. Phys. Lett. 69, 1208 (1996).

${ }^{5}$ J. Lu, G. Ravichandran, and W. L. Johnson, Acta Mater. 51, 3429 (2003).

${ }^{6}$ J. Schroers, Adv. Mater. 22, 1566 (2010).

${ }^{7}$ T. Ichitsubo, E. Matsubara, T. Yamamoto, H. S. Chen, N. Nishiyama, J. Saida, and K. Anazawa, Phys. Rev. Lett. 95, 245501 (2005).

${ }^{8}$ Y. L. Sun, D. D. Qu, Y. J. Sun, K. D. Liss, and J. Shen, J. Non-Cryst. Solids 356, 39 (2010).

${ }^{9}$ J. C. Ye, J. Lu, C. T. Liu, Q. Wang, and Y. Yang, Nature Mater. 9, 619 (2010).

${ }^{10}$ X. D. Wang, J. Bednarcik, K. Saksl, H. Franz, Q. P. Cao, and J. Z. Jiang, Appl. Phys. Lett. 91, 081913 (2007).

${ }^{11}$ X. D. Wang, J. Bednarcik, H. Franz, H. B. Lou, Z. H. He, Q. P. Cao, and J. Z. Jiang, Appl. Phys. Lett. 94, 011911 (2009).

${ }^{12}$ Y. Q. Cheng, E. Ma, and H. W. Sheng, Phys. Rev. Lett. 102, 245501 (2009).

${ }^{13}$ S. Plimpton, J. Comput. Phys. 117, 1 (1995).

${ }^{14}$ H. F. Poulsen, J. A. Wert, J. Neuefeind, V. Honkimaki, and M. Daymond, Nature Mater. 4, 33 (2005).

${ }^{15}$ T. C. Hufnagel, R. T. Ott, and J. Almer, Phys. Rev. B 73, 064204 (2006).

${ }^{16}$ Q. K. Jiang, X. D. Wang, X. P. Nie, G. Q. Zhang, H. Ma, H. J. Fecht, J. Bendnarcik, H. Franz, Y. G. Liu, Q. P. Cao, and J. Z. Jiang, Acta Mater. 56, 1785 (2008).

${ }^{17}$ Q. K. Li and M. Li, Intermetallics 14, 1005 (2006).

${ }^{18}$ Y. F. Shi, M. B. Katz, H. Li, and M. L. Falk, Phys. Rev. Lett. 98, 185505 (2007).

${ }^{19}$ Y. Q. Cheng and E. Ma, Prog. Mater. Sci. 56, 379 (2011).

${ }^{20}$ F. Spaepen, Acta Metall. 25, 407 (1977).

${ }^{21}$ L. Y. Chen, Z. D. Fu, G. Q. Zhang, X. P. Hao, Q. K. Jiang, X. D. Wang, Q. P. Cao, H. Franz, Y. G. Liu, H. S. Xie, S. L. Zhang, B. Y. Wang, Y. W. Zeng, and J. Z. Jiang, Phys. Rev. Lett. 100, 075501 (2008).

${ }^{22}$ A. I. Taub and F. Spaepen, Acta Metall. 28, 1781 (1980).

${ }^{23}$ M. Heggen, F. Spaepen, and M. Feuerbacher, J. Appl. Phys. 97, 033506 (2005).

${ }^{24}$ J. Li, Z. L. Wang, and T. C. Hufnagel, Phys. Rev. B 65, 144201 (2002).

${ }^{25}$ P. Murali, T. F. Guo, Y. W. Zhang, R. Narasimhan, Y. Li, and H. J. Gao, Phys. Rev. Lett. 107, 215501 (2011).
} 